\title{
Effects of PCV7 and PCV13 on invasive pneumococcal disease and carriage in Stockholm, Sweden
}

\author{
Ilias Galanis ${ }^{1,10}$, Ann Lindstrand ${ }^{1,2,10}$, Jessica Darenberg ${ }^{1,10}$, Sarah Browall ${ }^{3,4}$, \\ Priyanka Nannapaneni ${ }^{3}$, Karin Sjöström ${ }^{1}$, Eva Morfeldt ${ }^{1}$, Pontus Naucler ${ }^{5,6}$, \\ Margareta Blennow ${ }^{7,8}$, Åke Örtqvist ${ }^{5,9,11}$ and Birgitta Henriques-Normark ${ }^{1,3,4,11}$
}

\begin{abstract}
Affiliations: ${ }^{1}$ Public Health Agency of Sweden, Solna, Sweden. ${ }^{2}$ Dept of Public Health Sciences, Karolinska Institutet, Stockholm, Sweden. ${ }^{3}$ Dept of Microbiology, Tumor and Cell Biology, Karolinska Institutet, Stockholm, Sweden. ${ }^{4}$ Dept of Clinical Microbiology, Karolinska University Hospital, Stockholm, Sweden. ${ }^{5}$ Dept of Medicine Solna, Infectious Diseases Unit, Karolinska Institutet, Stockholm, Sweden. ${ }^{6}$ Dept of Infectious Diseases, Karolinska University Hospital, Stockholm, Sweden. ${ }^{7}$ Sachs' Children's Hospital, South General Hospital, Stockholm, Sweden. ${ }^{8}$ Dept of Clinical Sciences and Education, Karolinska Institutet, Stockholm, Sweden. ${ }^{9}$ Dept of Clinical Science and Education Södersjukhuset, Karolinska Institutet, Stockholm, Sweden. ${ }^{10}$ These authors contributed equally to this article. ${ }^{11}$ These authors contributed equally to this article.
\end{abstract}

Correspondence: Birgitta Henriques-Normark, Karolinska Institutet, MTC, 17177 Stockholm, Sweden. E-mail: birgitta.henriquesaki.se

ABSTRACT The effects of pneumococcal conjugated vaccines (PCVs) need to be investigated. In Stockholm County, Sweden, PCV7 was introduced in the childhood immunisation programme in 2007 and changed to PCV13 in 2010.

Over $90 \%$ of all invasive isolates during 2005-2014 $(\mathrm{n}=2336)$ and carriage isolates, 260 before and 647 after vaccine introduction, were characterised by serotyping, molecular typing and antibiotic susceptibility, and serotype diversity was calculated. Clinical information was collected for children and adults with invasive pneumococcal disease (IPD).

The IPD incidence decreased post-PCV7, but not post-PCV13, in vaccinated children. Beneficial herd effects were seen in older children and adults, but not in the elderly. The herd protection was more pronounced post-PCV7 than post-PCV13. PCV7 serotypes decreased. IPD caused by PCV13 serotypes 3 and 19A increased post-PCV7. Post-PCV13, serotypes 6A and 19A, but not serotype 3, decreased. The serotype distribution changed in carriage and IPD to nonvaccine types, also in nonvaccinated populations. Expansion of non-PCV13 serotypes was largest following PCV13 introduction. Serotype diversity increased and nonvaccine clones emerged, such as CC433 (serotype 22F) in IPD and CC62 (serotype 11A) in carriage. In young children, meningitis, septicaemia and severe rhinosinusitis, but not bacteraemic pneumonia, decreased.

Pneumococcal vaccination leads to expansion of new or minor serotypes/clones, also in nonvaccinated populations.

@ERSpublications

New or minor serotypes/clones expanded, also in nonvaccinated populations, affecting future vaccine strategies http://ow.ly/VA9EO

This article has supplementary material available from erj.ersjournals.com

Received: Aug 312015 | Accepted after revision: Dec 012015 | First published online: Feb 042016

Support statement: This work was supported by grants from the Swedish Research Council, the Stockholm County Council (ALF grant), the Swedish Foundation for Strategic research (SSF), and Knut and Alice Wallenberg foundation. Funding information for this article has been deposited with FundRef.

Conflict of interest: None declared.

Copyright OERS 2016. ERJ Open articles are open access and distributed under the terms of the Creative Commons Attribution Non-Commercial Licence 4.0. 


\section{Introduction}

Streptococcus pneumoniae is a major contributor to morbidity and mortality worldwide, and is the major cause of community-acquired pneumonia, with bacteraemia present in $<15 \%$ of adult pneumonia cases in some studies [1-3]. Pneumococci also cause meningitis with a high risk for sequelae and lethality $[4,5]$. Despite being a devastating pathogen, pneumococci are common colonisers of the upper respiratory tract of children [6]. Risk groups for clinical disease include very young children and the elderly, immunocompromised patients, patients with previous influenza virus infections, and patients with comorbidities, such as chronic lung disease, heart disease, malignancies and diabetes [7, 8].

Current pneumococcal vaccines are directed against a limited number of the so far described 97 serotypes, either as a polysaccharide-based 23-valent vaccine, or as protein-conjugated vaccines directed against seven (PCV7), 10 (PCV10) or 13 (PCV13) serotypes [9-19]. PCV7 was introduced in the USA in 2000 and since then many countries have introduced PCVs into their childhood vaccination programmes [20, 21]. A dramatic decrease of invasive pneumococcal disease (IPD) was observed after vaccine introduction in vaccinated children and a herd immunity effect was seen in other age groups [22-24]. However, the effect has been hampered by an increase of nonvaccine types (NVTs) [25]. Some studies show an increase in antibiotic-resistant NVTs, such as serotype 19A, post-PCV7, which might be a challenge for treatment [25, 26]. During 2009-2011, PCV7 was replaced by PCV10 or PCV13 and an additional effect of PCV13 was observed in some countries [24, 27-29]. However, more data are needed on changes in clinical outcome and the effects of PCV7 and PCV13, respectively, on serotype diversity and clonal types of strains causing IPD in all age groups and carriage from the same geographic area.

In Sweden it is mandatory to report IPD cases to the Public Health Agency of Sweden. In Stockholm County, PCV7 was introduced into the child vaccination programme in October 2007, given in a $2+1$ schedule, and replaced with PCV13 in January 2010. The vaccine coverage in Stockholm is close to $97 \%$ (for 2-year-old children born in 2011 in Stockholm) (http://www.folkhalsomyndigheten.se/pagefiles/21426/ Arsrapport-barnvaccinationsprogramet-2014-15032.pdf). This population-based study describes the effects of PCV7 introduction and the replacement with PCV13 in Stockholm, Sweden. The incidence of IPD and carriage, as well as clinical outcome, serotype and clonal distribution, and antibiotic resistance, were studied 3 years before vaccine introduction and 7 years after PCV introduction (2 years of PCV7 use and 4 years of PCV13 use).

\section{Materials and methods}

\section{Clinical pneumococcal isolates}

Pneumococcal isolates $(\mathrm{n}=2336$, representing $93 \%$ of all 2519 reported cases) from patients $0-<2,2-<18$, $18-<65$ and $\geqslant 65$ years old $(65-<80$ and $\geqslant 80$ years old), with IPD (pneumococci recovered from blood, cerebrospinal fluid or other sterile sites) in Stockholm County were collected from the clinical microbiological laboratories during 2005-2014. Also see online supplementary material.

Pneumococcal carriage isolates before vaccine introduction $(n=260)$ were collected with nasopharyngeal swabs from 719 children between 1 and 5 years old (mean age 3 years) attending day-care centres in Stockholm during 2004. After vaccine introduction, nasopharyngeal aspirates were collected from 2161 healthy children $0-<6$ years old (mean age 1 year) at regular child healthcare visits in Stockholm during 2011-2013, yielding 647 pneumococcal isolates. Also see online supplementary material.

The study was approved by the Regional Ethical Committee in Stockholm County.

\section{Clinical information}

Clinical information for all reported IPD cases in children $<18$ years of age $(n=161)$ during the study period 2005-2014 was collected retrospectively using medical records. Clinical data were collected for patients $\geqslant 18$ years of age during $2007-2013$ using clinical questionnaires $(n=986)$.

\section{Serotyping}

All pneumococcal isolates were serotyped by gel diffusion and/or Quellung reactions as previously described [30].

\section{Molecular typing methods}

Pulsed-field gel electrophoresis as adapted from the procedure described by Herman et al. [31] and multilocus sequence typing were performed on selected isolates as described previously [32], see online supplementary material. 
Antibiotic susceptibility testing

Antibiotic susceptibility was tested for penicillin G using discs and Etest as described elsewhere [33]. Isolates were classified according to the European Committee on Antimicrobial Susceptibility Testing system of species-related breakpoints (nonmeningitis) (www.eucast.org).

\section{Statistical analyses}

The incidence rate ratios of IPD were calculated pairwise to enable comparisons between the pre-vaccination years (2005-2007), after PCV7 vaccine introduction (2009-2010) and post-PCV13 (2011-2014). Confidence intervals and p-values were calculated based on a normal approximation or Poisson exact test. The annual number of inhabitants by age group was obtained from Statistics Sweden (www.scb.se). Missing serotypes were imputed as described in the online supplementary material. The serotype diversity was measured with the Simpson index of diversity as described in the online supplementary material.

Chi-squared and Wilcoxon rank-sum tests were used to compare variables between two periods, while Holm's method of adjustment for multiple comparison was applied when appropriate. Two-sided p-values $<0.05$ were considered to be statistically significant. The statistical software R (version 3.2.0; www.r-project. org), and packages "epitools" (version 0.5-7) and "exactci" (version 1.3-0) were used.

\section{Results}

\section{Effects on IPD incidence after PCV7 introduction and its replacement by PCV13}

PCV7 introduction in 2007 led to a slight decrease of IPD incidence in the entire population (18\%, 95\% CI $8-27$ ), that was followed by a further reduction after PCV13 was introduced in 2010 (11\%, 95\% CI 1-21). Hence, the total incidence went from 16 cases in 2005 to nine cases in 2014 per 100000 population (figure 1a) due to a dramatic decrease of PCV7 type strains and a modest decrease of the extra six serotypes in PCV13 post-PCV13. However, non-PCV13 strains increased (figure 1b). The rate ratio for IPD in different age groups was calculated and compared for three time periods: before PCV7 was introduced (2005-2007), post-PCV7 (2009-2010) and post-PCV13 (2011-2014) (online supplementary tables E1-E6).

For the youngest children $<2$ years of age, there was a significant decrease when comparing post-PCV7 and post-PCV13 with the pre-vaccine period, but not when comparing post-PCV13 with post-PCV7 (figure $2 \mathrm{a}$ and online supplementary table E1). However, the six extra serotypes in PCV13 decreased when comparing post-PCV7 with post-PCV13 and there were no cases of IPD caused by PCV13 types during the last 2 years of this study, suggesting protection also against these types. Importantly, the incidence of non-PCV13 strains was doubled following PCV13 introduction.

For children $2-<18$ years of age, the IPD incidence was low even before vaccine introduction, but a beneficial effect was nevertheless seen (figure $2 \mathrm{~b}$ and online supplementary table E2). Thus, there were no IPD cases caused by PCV7 types from 2011.

In the nonvaccinated group $18-<65$ years of age, there was a reduction of IPD incidence post-PCV7 that became more pronounced post-PCV13 (figure $2 \mathrm{c}$ and online supplementary table E3).
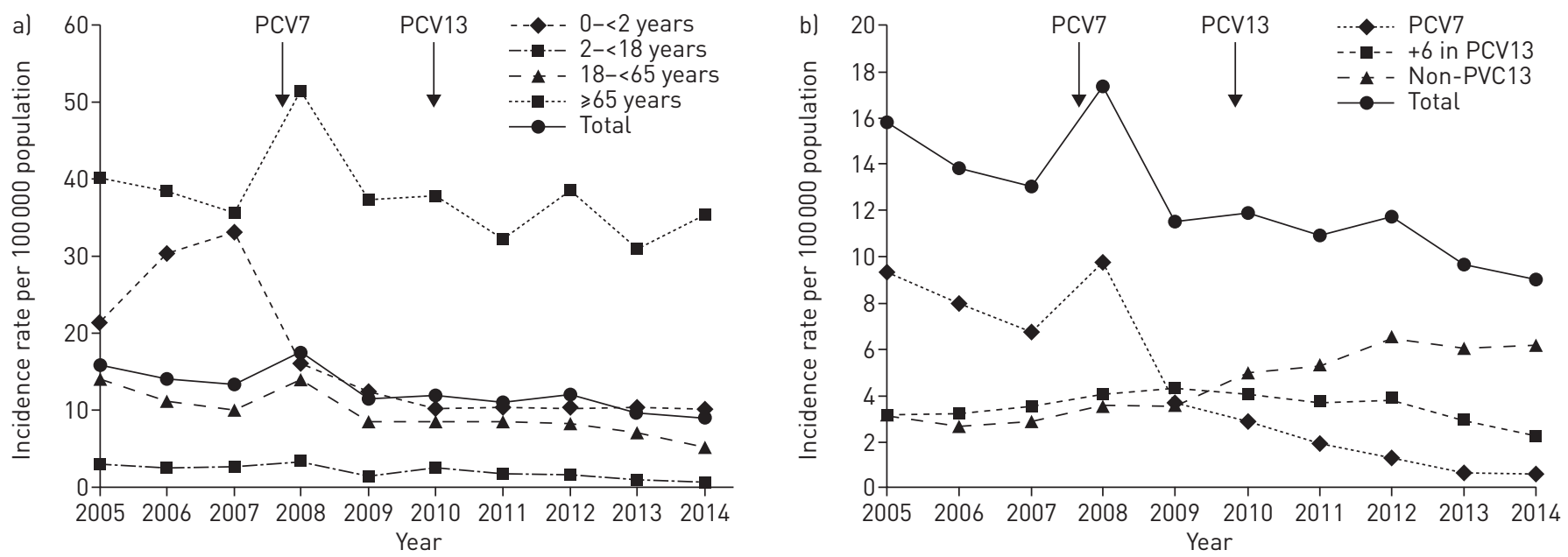

FIGURE 1 a) Incidence rates of invasive pneumococcal disease (IPD) in different age groups during 2005-2014. b) Incidence rates of vaccine and nonvaccine type IPD during 2005-2014. The arrows indicate the introduction of pneumococcal conjugated vaccine PCV7 and the change to PCV13 in the childhood vaccination programme. 

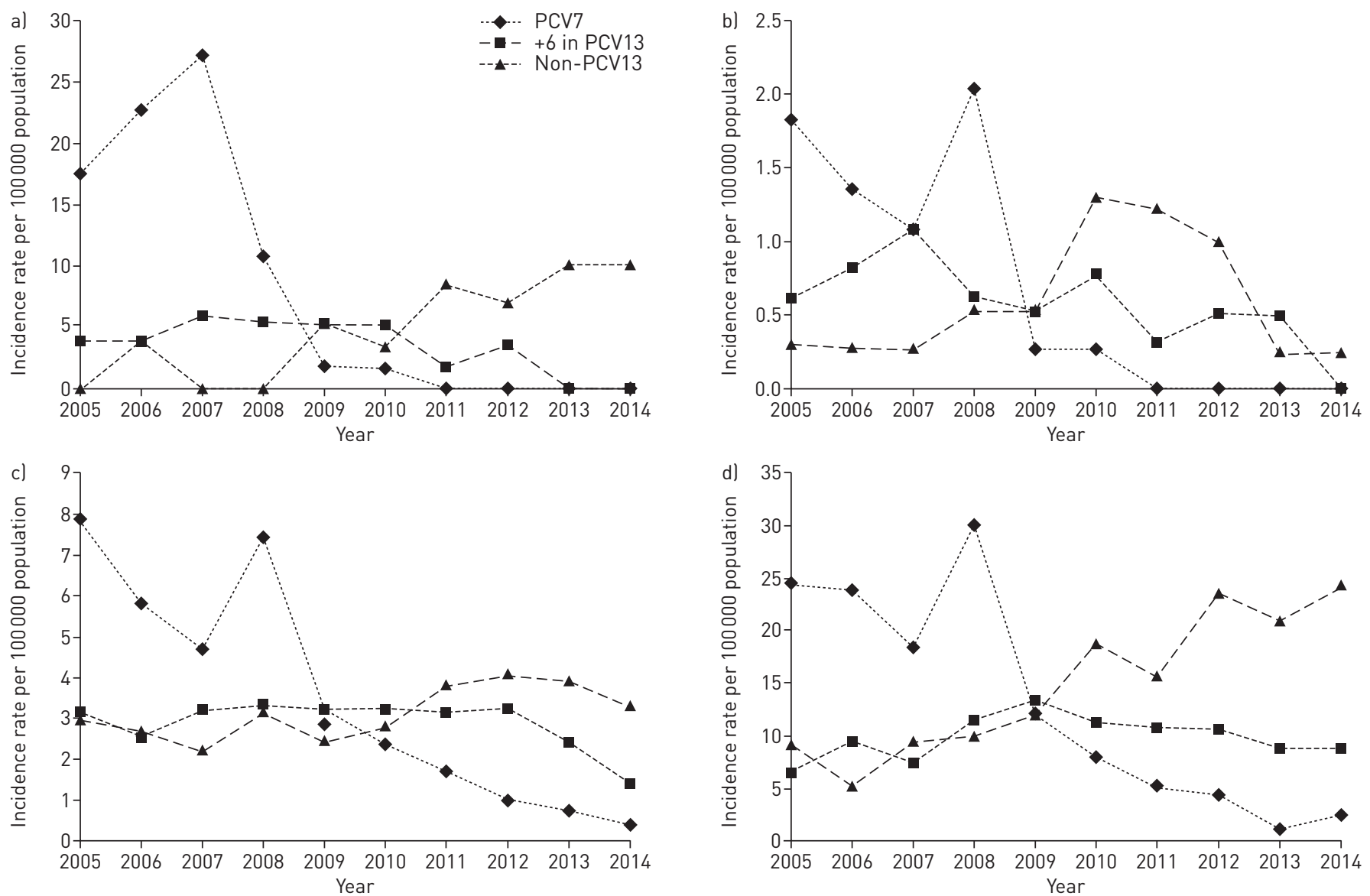

FIGURE 2 Serotype-specific incidence in a) the youngest children aged $<2$ years, b) children $2-<18$ years, c) adults $18-<65$ years and d) the elderly aged $\geqslant 65$ years.

In the oldest age group $\geqslant 65$ years, there was no significant decrease of the overall IPD incidence post-PCV (figure $2 \mathrm{~d}$ and online supplementary table E4). Even though PCV7 types diminished significantly, especially post-PCV13, this was counteracted by the large increase of non-PCV13 strains during both the post-PCV7 and post-PCV13 periods. However, the six extra serotypes present in PCV13, which increased post-PCV7, tended to decrease post-PCV13. Non-PCV13 strains were more common in the $\geqslant 80$ years age group than in the 65-<80 years age group (online supplementary tables E5 and E6, and online supplementary figures E1 and E2).

Comparing individual serotypes causing IPD before and post-PCV7 or post-PCV13

Rate ratios for individual serotypes were compared for the three time periods (figures 3 and 4). Most PCV7 serotypes decreased significantly post-PCV7 compared with pre-PCV except for serotypes 4 and 18C (figure 3a), although these two serotypes decreased post-PCV13 (figure 3b). Among the six extra serotypes in PCV13, only serotype 7F was significantly reduced post-PCV13 compared with pre-PCV and post-PCV7 (figure $3 \mathrm{~b}$ and $\mathrm{c}$ ). However, there was a tendency for decreased rate ratios, albeit not significant, for serotypes $6 \mathrm{~A}$ and $19 \mathrm{~A}$ post-PCV13 compared with post-PCV7. Serotype 3 increased significantly post-PCV7 compared with pre-PCV, but there was no change when comparing post-PCV13 with post-PCV7. Non-PCV13 serotype 22F increased significantly post-PCV7 compared with pre-vaccination, and serotypes $10 \mathrm{~A}$ and $23 \mathrm{~A}$ increased post-PCV13 compared with post-PCV7 (figure 4). In addition, several less abundant non-PCV13 serotypes increased significantly post-PCV13 compared with post-PCV7. Expansion of non-PCV13 serotypes was largest following PCV13 introduction.

\section{Expansion of nonvaccine pneumococcal serotypes in carriage after vaccine introduction}

Pneumococcal carriage rates in healthy children were 36\% before and 30\% after vaccine introduction $(\mathrm{p}=0.002)$. Before vaccination, $38 \%$ and $18 \%$ of carriage isolates represented non-PCV7 and non-PCV13 types, respectively, and the corresponding values after vaccination were $95 \%$ and $89 \%$, respectively. The serotype distribution changed dramatically, with PCV7 serotypes $19 \mathrm{~F}$ and $6 \mathrm{~B}$ and PCV13 serotype $6 \mathrm{~A}$ 
a)

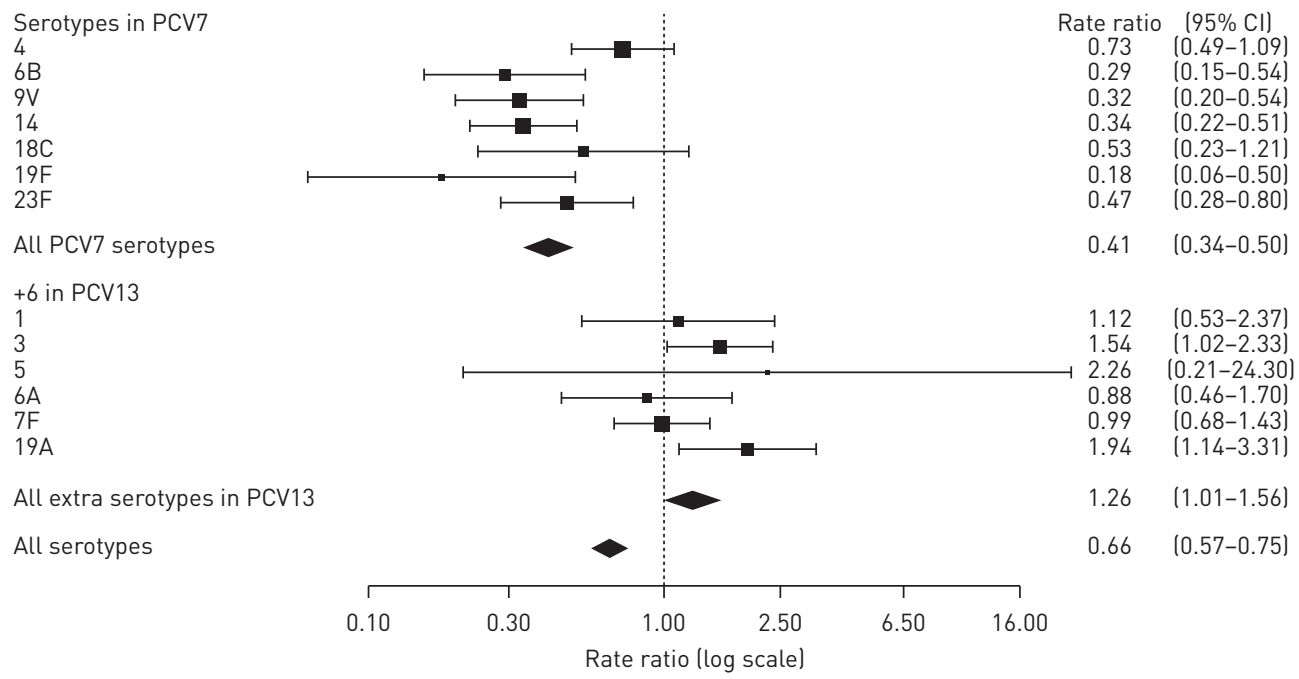

b)

Serotypes in PCV7
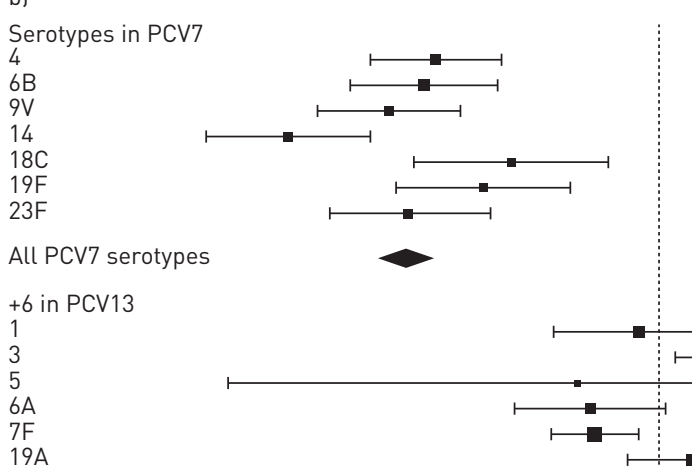

Rate ratio $(95 \% \mathrm{CI})$

$0.17 \quad(0.10-0.29)$

$\begin{array}{ll}0.16 \quad(0.09-0.29) \\ 0.16 & (0.07-0.21)\end{array}$

$0.12 \quad(0.07-0.21)$

0.06 (0.03-0.11)

$0.32 \quad(0.15-0.68)$

$0.25 \quad(0.13-0.49)$

$0.14 \quad(0.08-0.26)$

$0.14 \quad(0.11-0.17)$

All PCV7 serotypes

$0.87 \quad(0.44-1.69)$

$1.62 \quad(1.13-2.32)$

$0.53 \quad(0.03-8.31)$

$0.59 \quad(0.32-1.07)$

$\begin{array}{ll}0.59 & (0.32-1.07) \\ 0.61 & (0.43-0.85)\end{array}$

$1.31(0.79-2.16)$

All extra serotypes in PCV13

0.97 (0.80-1.17)

All serotypes

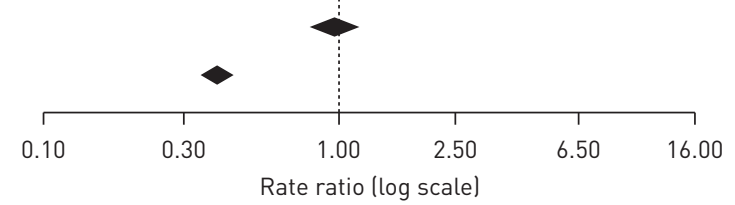

$0.38 \quad(0.34-0.44)$

c)

Serotypes in PCV7

6B

$9 \mathrm{~V}$

$14 \mathrm{C}$

$19 \mathrm{~F}$

23F

All PCV7 serotypes

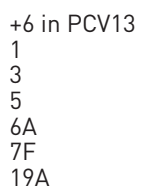

All extra serotypes in PCV13

All serotypes
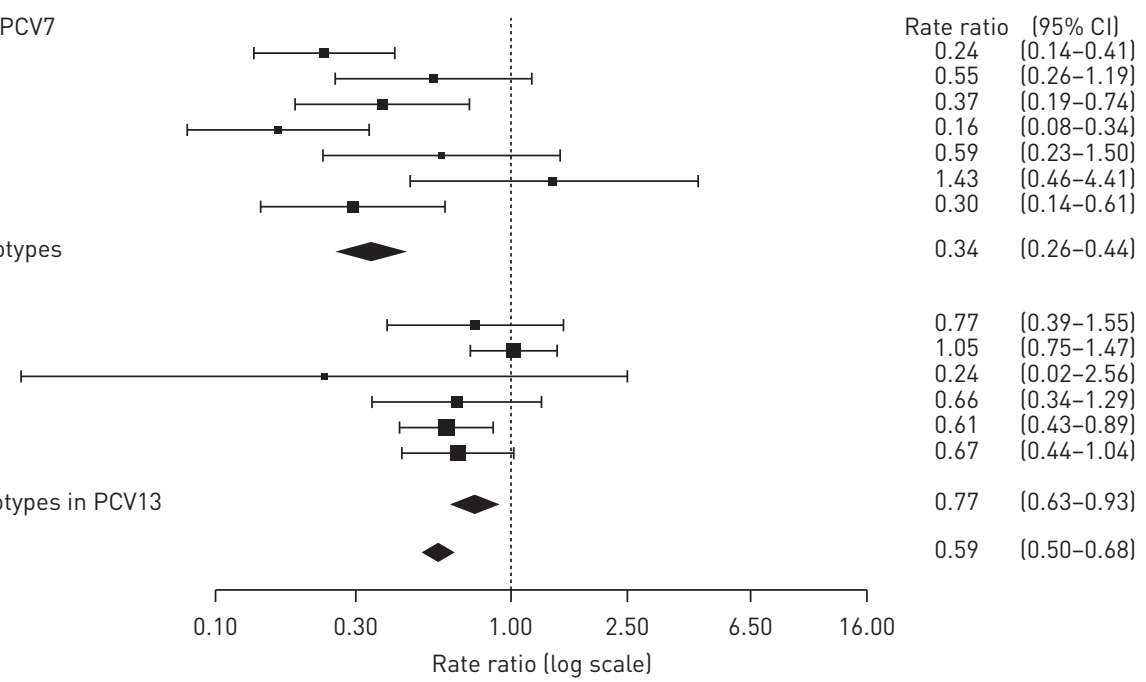

FIGURE 3 Rate ratios for serotypes included in pneumococcal conjugated vaccines PCV7 and PCV13 comparing al post-PCV7 with pre-vaccination, b) post-PCV13 with pre-vaccination and c) post-PCV13 with post-PCV7. 


$$
\text { a) }
$$

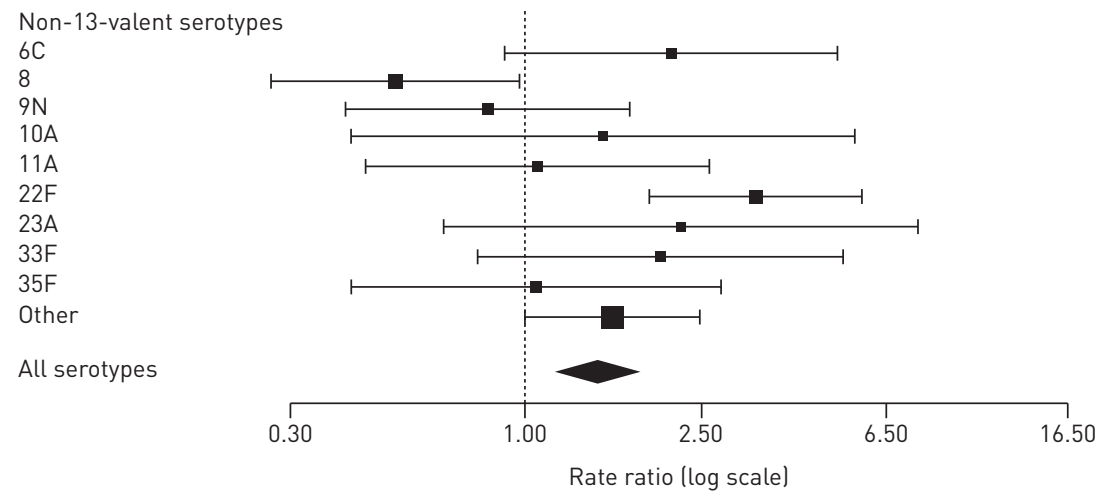

Rate ratio $(95 \% \mathrm{CI})$

$2.13 \quad(0.90-5.04)$

$0.52 \quad(0.27-0.98$

$0.82 \quad(0.40-1.71)$

$1.51 \quad(0.41-5.55)$

$1.07 \quad(0.44-2.63)$

$3.29 \quad(1.90-5.71)$

$2.26 \quad(0.66-7.66)$

$2.02 \quad(0.79-5.18)$

1.07 (0.41-2.75)

$1.58(1.01-2.48)$

1.45 (1.17-1.81)

,

Non-13-valent serotypes

$6 \mathrm{C}$

8

$9 \mathrm{~N}$

$10 \mathrm{~A}$

$11 \mathrm{~A}$
$22 \mathrm{~F}$

23A

$33 \mathrm{~F}$

$35 \mathrm{~F}$

Other

All serotypes

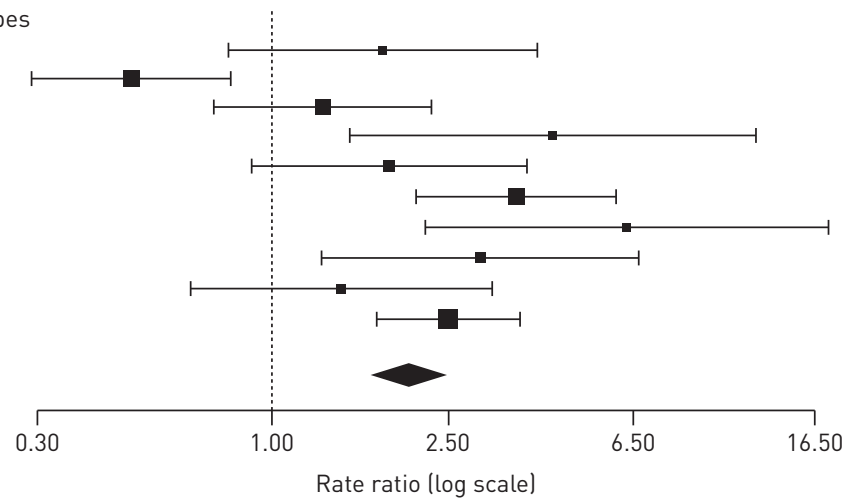

Rate ratio $(95 \% \mathrm{CI})$

$1.81 \quad(0.82-3.97)$

$0.50 \quad(0.30-0.84)$

$1.33 \quad(0.77-2.30)$

$4.27 \quad(1.52-11.99)$

$1.87 \quad(0.93-3.77)$

$3.54 \quad(2.14-5.87)$

$6.26 \quad(2.26-17.37)$

$2.97 \quad(1.32-6.65)$

$1.46 \quad(0.68-3.13)$

$2.53 \quad(1.74-3.67)$

$2.06 \quad \mid 1.71-2.47$

0.30

Rate ratio (log scale)

c)

Non-13-valent serotypes 6C

8

$9 \mathrm{~N}$

$10 \mathrm{~A}$
$11 \mathrm{~A}$

$22 \mathrm{~F}$

23A

$33 \mathrm{~F}$

$35 \mathrm{~F}$

Other

All serotypes

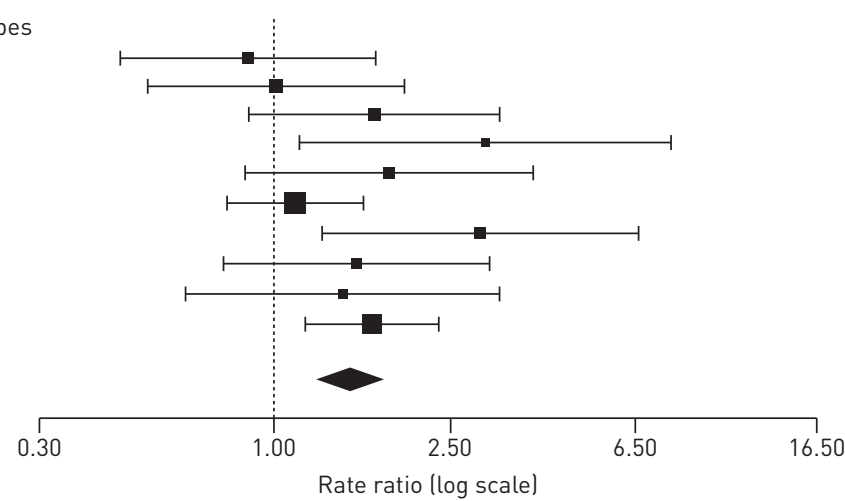

FIGURE 4 Rate ratios for non-PCV13 pneumococcal conjugated vaccine serotypes comparing al post-PCV7 with pre-vaccination, b) post-PCV13 with pre-vaccination and c) post-PCV13 with post-PCV7.

dominating before vaccine introduction, and non-PCV13 serotypes $11 \mathrm{~A}, 35 \mathrm{~F}, 23 \mathrm{~A}, 21,15 \mathrm{~B}$ and $23 \mathrm{~B}$ dominating after vaccine introduction (figure 5). Serotypes in PCV7 and serotypes 6A and 3 included in PCV13 decreased, but several previously minor non-PCV13 serotypes expanded after vaccination. This resulted in increased serotype diversity as measured by the Simpson index (table 1).

Increased diversity of serotypes in IPD after vaccine introduction

Some non-PCV13 serotypes, such as 22F, increased shortly after PCV7 introduction in IPD. Serotypes 23A and $23 \mathrm{~B}$ increased later, suggesting that they emerged as a consequence of PCV13 vaccination. The distribution of serotypes in IPD reflected largely the carriage data (figure 5). However, even though PCV13 serotypes 3 and 19A, and non-PCV13 serotypes $22 \mathrm{~F}$ and $23 \mathrm{~A}$, showed the largest increase post-vaccination in IPD (figure 6), serotypes $11 \mathrm{~A}$ and $35 \mathrm{~F}$ dominated in vaccinated carriers (figure 5). IPD patients infected with serotype 11A were significantly older than those with PCV13 serotypes 1, 4, 7F, 8, $9 \mathrm{~V}, 14$ or $18 \mathrm{C}$ (data not shown). 


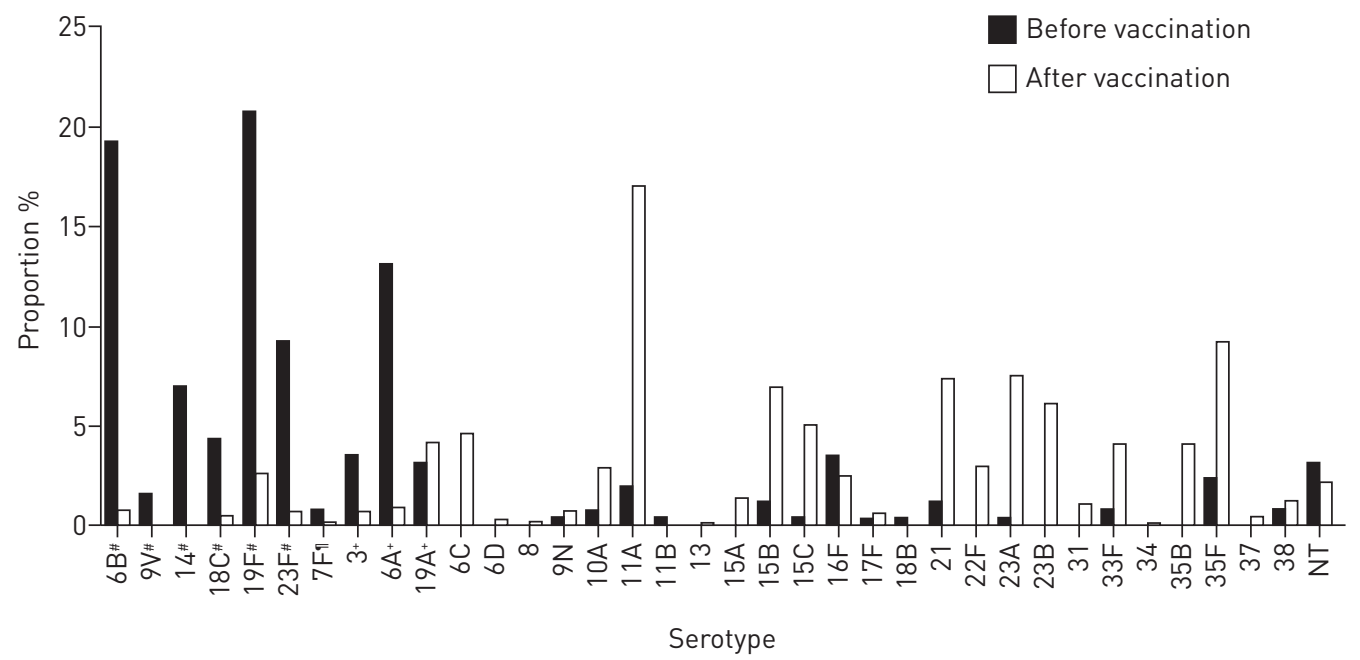

FIGURE 5 Proportion of serotypes in carriage in children before $(n=260)$ and after $(n=647)$ pneumococcal conjugated vaccine introduction. NT: nontypeable. ": serotypes included in PCV7; ": additional serotypes included in PCV10; ${ }^{+}$: additional serotypes included in PCV13.

The Simpson index of diversity for IPD was significantly higher after than before vaccine introduction (table 1) and the Simpson index peaked in 2011 post-PCV13 (online supplementary table E7).

Clonal selection of PCV13 serotype 3 and non-PCV13 serotype 22F post-vaccination in IPD, and of non-PCV13 serotype $11 \mathrm{~A}$ post-vaccination in carriage

The unexpected post-PCV7 increase of serotype 3 that prevailed post-PCV13 was due to an expansion of two pre-existing genetic lineages (CC180 and CC378), accounting for $72 \%$ and $18 \%$ of serotype 3 isolates, respectively, in 2012 (data not shown).

Before vaccine introduction there were no non-PCV13 serotype $22 \mathrm{~F}$ isolates in carriage and only a few caused IPD, with those isolates belonging to two different clones, i.e. CC433 and CC1012 (data not shown). The significantly increased incidence of serotype $22 \mathrm{~F}$ post-PCV was solely due to an expansion of one of these genetic lineages, i.e. CC433 (data not shown).

Expansion of serotype 11A in childhood carriage was mainly due to an increase of clonal type CC62 (data not shown), a clonal type also found in IPD.

Resistance to penicillin G among IPD cases before and after vaccine introduction

There was no statistical difference in the overall susceptibility to penicillin $G$ before and after vaccine introduction among IPD isolates (4.4\% pre-PCV versus $4.8 \%$ post-PCV7 and $6.0 \%$ post-PCV13). However, PCV7 serotypes changed, where $5.5 \%$ of the isolates pre-PCV, $7.1 \%$ post-PCV7 and $26.1 \%$ post-PCV 13 had a reduced susceptibility to penicillin $G(p<0.001)$. No differences in susceptibility were found for non-PCV13 serotypes in IPD during the three time periods (data not shown).

Clinical presentation in children before and after vaccine introduction

We compared the clinical presentation in children <2 years before (2005-2007) and after vaccine introduction (2009-2014) (table 2). The incidence rates of rhinosinusitis, meningitis and septicaemia decreased significantly post-PCV, while the incidence of pneumonia as a cause of IPD remained unaltered. The proportion of children with underlying comorbidities increased from $40 \%$ to $43 \%$ post-PCV, with the

TABLE 1 Serotype diversity in invasive pneumococcal disease (IPD) and carriage before and after vaccine introduction calculated using the Simpson index of diversity

\begin{tabular}{ccc}
\multicolumn{2}{c}{ Simpson index $(95 \% \mathrm{Cl})$} & p-value \\
\cline { 1 - 2 } Before & After & \\
$0.928(0.921-0.935)$ & $0.946(0.942-0.950)$ & $<0.001$ \\
$0.885(0.866-0.904)$ & $0.930(0.922-0.938)$ & $<0.001$
\end{tabular}



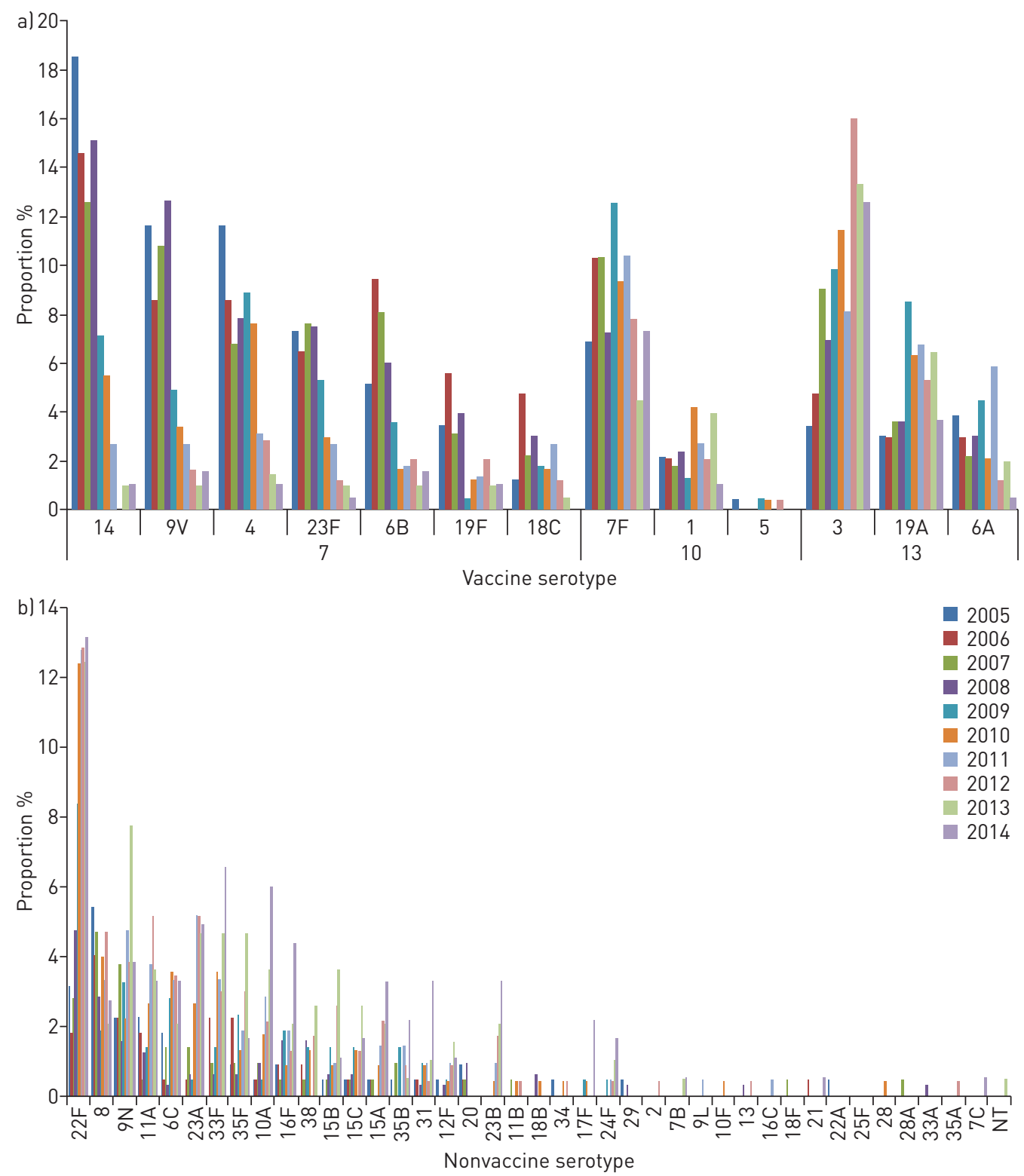

FIGURE 6 Proportion of serotypes in invasive pneumococcal disease in all ages during 2005-2014: a) vaccine serotypes and b) nonvaccine serotypes. NT: nontypeable.

TABLE 2 Clinical presentation of invasive pneumococcal disease in children $0-<2$ years before (2005-2007) and after (2008-2014) vaccine introduction

\begin{tabular}{lcccc} 
& \multicolumn{2}{c}{ Incidence rate } & Rate ratio $(95 \%$ Cl) & p-value \\
\cline { 2 - 3 } & Before & After & & \\
\hline Meningitis & $10.1(16)$ & $4.3(15)$ & $0.43(0.21-0.86)$ & 0.02 \\
Septicaemia & $6.3(10)$ & $2.0(7)$ & $0.32(0.12-0.84)$ & 0.02 \\
Pneumonia & $3.8(6)$ & $2.9(10)$ & $0.76(0.28-2.09)$ & 0.59 \\
Rhinosinusitis & $5.0(8)$ & $0.6(2)$ & $0.11(0.02-0.54)$ & 0.006 \\
Other & $3.2(5)$ & $0.9(3)$ & $0.27(0.07-1.14)$ & 0.08 \\
Total & $28.4(45)$ & $10.6(37)$ & $0.37(0.24-0.58)$ & $<0.001$ \\
\hline
\end{tabular}

Data are presented as $\mathrm{n}$ or rate per 100000 person-years (n), unless otherwise stated. ${ }^{\text {: }}$ bacteraemia without known focus, mastoiditis, osteomyelitis, septic arthritis and cellulitis. 
most common comorbidities being chronic lung disease and neurological disease. Based on mean days of treatment, percentage treated in the intensive care unit and clinical presentation, including parameters such as fever, heart rate, respiratory rate, C-reactive protein, haemoglobin and leukocyte count, we found no significant differences in severity of IPD after vaccine introduction (data not shown). The mean age among the youngest children with IPD was 1.04 years pre-PCV, while it was 0.88 years post-PCV $(\mathrm{p}=0.18)$. More boys than girls $(56 \%$ versus $44 \%)$ presented with an IPD in the pre-PCV period and this difference increased post-PCV (65\% boys, $\mathrm{p}=0.53)$.

In the age group $2-<5$ years, the proportion with underlying comorbidities remained stable around $73-75 \%$; however, in the age group 5-<18 years, the proportion of comorbidities increased from $69 \%$ to $80 \%$. The main comorbidities in older children were neurologic disease and malignancies. Pneumonia decreased in both age groups (online supplementary tables E8 and E9).

\section{Clinical presentation in other age groups}

To study whether the clinical presentation changed in other age groups we used clinical questionnaires completed by clinicians for patients $\geqslant 18$ years of age starting in 2007 (online supplementary tables E10E12). The response rate was $54 \%$ in 2007 and $79 \%$ in $2009-2013$. We observed a decrease of septicaemia in the age group $65-<80$ years.

\section{Discussion}

In this population-based study we examined the impact of PCV7 introduction in late 2007 and its replacement by PCV13 in early 2010 in Stockholm County, Sweden, an area with around 2 million inhabitants.

The IPD incidence in children $<2$ years of age decreased significantly post-PCV7. Also, no further cases of IPD were caused by PCV13 serotypes during the final years of this study, arguing that PCV13 provides protection against all serotypes included. Children with IPD post-PCV tended to be younger and more often to be boys. Although the incidence of pneumonia as a cause of IPD was not reduced, we found a $19 \%$ lower risk for hospitalisation in children with pneumonia after vaccine introduction in a previous study [34].

The IPD incidence did not decrease significantly in the elderly post-PCV. However, for all other age groups, and overall, there was a small decrease post-PCV13, which is in concordance with other studies [24, 27-29]. This was mainly due to a gradual decrease of IPD caused by the six extra serotypes in PCV13, and a low incidence of non-PCV13 strains in the age groups $2-<18$ and $18-<65$ years. However, due to expansion of non-PCV13 strains, no large beneficial effect on the IPD incidence was observed by replacing PCV7 with PCV13 for the youngest children or for the elderly. The herd protection effects post-PCV13 were less pronounced as compared with the effects post-PCV7. In addition, in the elderly, the incidence of IPD caused by the six extra serotypes in PCV13 remained high post-PCV13. This suggests that it may take a longer time before the full herd effect can be observed in this population. Despite this, our results indicate that only a limited effectiveness of vaccination of the elderly with PCV13 can be expected, since already 5 years after PCV13 introduction $68 \%$ of IPD cases among the elderly were caused by non-PCV13 strains. However, the incidence of PCV13 serotypes as a cause of IPD in the elderly was about 15/100000 in the later years, mostly due to the six "new" serotypes added to PCV7, suggesting that vaccination with PCV13 still could be beneficial in this age group, at least in the near future.

All PCV7 serotypes decreased in IPD post-PCV7. A small decrease was also observed for the six extra serotypes in PCV13 in children $2-<18$ years of age. Post-PCV13, only serotype $7 \mathrm{~F}$ showed a significant decrease in incidence relative to post-PCV7, but the decrease in 2014 of serotypes 1, 6A and 19A suggests that IPD caused by most if not all extra serotypes in PCV13 will decrease in the years to come. A surprising finding was the expansion of serotype 3 preferentially among adults. Clonal analyses reveal that most of these isolates represent expansion of clone CC180 previously shown to be associated with a high mortality [10, 35]. Serotype 3 reached $16 \%$ of all IPD cases in 2012 and was still the most prevalent IPD serotype in Stockholm County in 2014. Our carriage data and a published report suggest that PCV13 might generate immunogenicity to serotype 3 [36]. One explanation for our findings could be that serotype 3 has another reservoir in addition to the nasopharynx of small children.

Some of the non-PCV13 serotypes had already expanded post-PCV7, such as serotype 22F, the most common NVT in 2014. Interestingly, a specific clone of type 22F, i.e. CC433, expanded post-PCV7, while another clone of the same serotype that was present pre-PCV vanished post-PCV. CC433 was not found pre-PCV among carriers, but was found in relatively low numbers in this group post-PCV. The abundance of CC433 in IPD relative to carriage suggest a relatively high invasive disease potential for this clone. 
A large number of other non-PCV13 types causing IPD increased post-PCV, largely reflecting the increased nasopharyngeal carriage of non-PCV13 strains post-vaccination. One example is serotype 11A that was the most common serotype in carriage post-PCV due to a large clonal expansion (CC62). Our previous studies have shown that serotype 11A causes IPD in patients with previous underlying diseases [10]. Here, IPD patients infected with serotype $11 \mathrm{~A}$ were significantly older than those infected by other vaccine type strains, arguing that risk factors need to be present for disease to occur for this clonal lineage.

Some non-PCV13 serotypes, such as 10A, 11A, 23A and 23B, increased in IPD preferentially during the post-PCV13 period. This likely reflects an altered nasopharyngeal composition of pneumococcal serotypes post-PCV13 compared with post-PCV7, but this assumption needs to be verified.

We observed that PCV introduction resulted in an almost complete replacement of vaccine types to NVTs in the nasopharynx of healthy children, without affecting carriage rates substantially. Since post-PCV carrier isolates were mainly isolated from PCV13-vaccinated children, we conclude that PCV13 provides carriage protection to most PCV13 serotypes during this time period, except for serotype 19A. The nasopharynx of small children represents the main source for pneumococcal transmission, explaining the significant increase of non-PCV13 strains in IPD. Interestingly, this increase was most pronounced for the youngest children and the elderly, i.e. age groups with the highest risk for developing IPD. This might indicate that expanding non-PCV13 strains are more prone to cause disease in individuals with risk factors as compared with many vaccine type strains.

In this study we characterised pneumococcal isolates available from $93 \%$ of all IPD cases in the study area during the study period. Hence, the coverage rate was nearly complete and we have no indications that secular trends such as changed sampling procedures or reporting rates could have influenced the outcome [37]. We cannot totally exclude that changes in comorbidities, antibiotic selection or intrinsic pneumococcal serotype trends could have influenced the serotype distribution, but it seems less likely that this plays a major role here. The elimination of vaccine type strains in healthy carriage will create profound changes in the entire pneumococcal population structure within a community since different pneumococcal strains most likely coevolve as a result of reciprocal adaptation and counter-adaptation between interacting strains. This represents the key force structuring biological diversity in different ecosystems as postulated in the Red Queen hypothesis [38]. Our findings here demonstrate that serotype diversity during carriage increases significantly as a result of PCV vaccination. The increased serotype diversity in IPD post-PCV is most likely a reflection of the increased number of serotypes prevailing in vaccinated carriers, which will influence the success of current vaccine strategies and must be taken into account when future strategies are developed.

\section{Acknowledgements}

We thank Ingrid Andersson, Gunnel Möllberg and Christina Johansson from the Swedish Institute for Infectious Disease Control and the Public Health Agency of Sweden, Kerstin Jämtberg and Carita Krokstrand from Sachs' Children and Youth Hospital, South General Hospital, Stockholm, Sweden, for excellent technical expertise, and the clinical microbiological laboratories for sending pneumococcal isolates.

\section{References}

1 Kalin M, Ortqvist A, Almela M, et al. Prospective study of prognostic factors in community-acquired bacteremic pneumococcal disease in 5 countries. J Infect Dis 2000; 182: 840-847.

2 O'Brien KL, Wolfson LJ, Watt JP, et al. Burden of disease caused by Streptococcus pneumoniae in children younger than 5 years: global estimates. Lancet 2009; 374: 893-902.

3 Pereira JM, Basílio C, Sousa-Dias C, et al. Can we predict pneumococcal bacteremia in patients with severe community-acquired pneumonia? J Crit Care 2013; 28: 970-974.

4 Koedel U, Scheld WM, Pfister HW. Pathogenesis and pathophysiology of pneumococcal meningitis. Lancet Infect Dis 2002; 2: 721-736.

5 Stockmann C, Ampofo K, Byington CL, et al. Pneumococcal meningitis in children: epidemiology, serotypes, and outcomes from 1997-2010 in Utah. Pediatrics 2013; 132: 421-428.

6 Henriques-Normark B, Tuomanen EI. The pneumococcus: epidemiology, microbiology, and pathogenesis. Cold Spring Harb Perspect Med 2013; 3: a010215.

$7 \quad$ McCullers JA, McAuley JL, Browall S, et al. Influenza enhances susceptibility to natural acquisition of and disease due to Streptococcus pneumoniae in ferrets. J Infect Dis 2010; 202: 1287-1295.

8 Klugman KP, Madhi SA. Pneumococcal vaccines and flu preparedness. Science 2007; 316: 49-50.

9 Sandgren A, Sjostrom K, Olsson-Liljequist B, et al. Effect of clonal and serotype-specific properties on the invasive capacity of Streptococcus pneumoniae. J Infect Dis 2004; 189: 785-796.

10 Sjostrom K, Spindler C, Ortqvist A, et al. Clonal and capsular types decide whether pneumococci will act as a primary or opportunistic pathogen. Clin Infect Dis 2006; 42: 451-459.

11 Bentley SD, Aanensen DM, Mavroidi A, et al. Genetic analysis of the capsular biosynthetic locus from all 90 pneumococcal serotypes. PLoS Genet 2006; 2: e31.

12 Bratcher PE, Kim KH, Kang JH, et al. Identification of natural pneumococcal isolates expressing serotype 6D by genetic, biochemical and serological characterization. Microbiology 2010; 156: 555-560. 
Calix JJ, Porambo RJ, Brady AM, et al. Biochemical, genetic, and serological characterization of two capsule subtypes among Streptococcus pneumoniae serotype 20 strains: discovery of a new pneumococcal serotype. J Biol Chem 2012; 287: 27885-27894.

14 Henrichsen J. Six newly recognized types of Streptococcus pneumoniae. J Clin Microbiol 1995; 33: 2759-2762.

15 Ko KS, Baek JY, Song JH. Capsular gene sequences and genotypes of "serotype 6E" Streptococcus pneumoniae isolates. J Clin Microbiol 2013; 51: 3395-3399.

16 Oliver MB, van der Linden MP, Küntzel SA, et al. Discovery of Streptococcus pneumoniae serotype 6 variants with glycosyltransferases synthesizing two differing repeating units. J Biol Chem 2013; 288: 25976-25985.

17 Park IH, Pritchard DG, Cartee R, et al. Discovery of a new capsular serotype (6C) within serogroup 6 of Streptococcus pneumoniae. J Clin Microbiol 2007; 45: 1225-1233.

18 Zartler ER, Porambo RJ, Anderson CL, et al. Structure of the capsular polysaccharide of pneumococcal serotype 11A reveals a novel acetylglycerol that is the structural basis for 11A subtypes. J Biol Chem 2009; 284: 7318-7329.

19 Lin FL, Vinogradov E, Deng C, et al. Structure elucidation of capsular polysaccharides from Streptococcus pneumoniae serotype 33C, 33D, and revised structure of serotype 33B. Carbohydr Res 2014; 383: 97-104.

20 Fitzwater SP, Chandran A, Santosham M, et al. The worldwide impact of the seven-valent pneumococcal conjugate vaccine. Pediatr Infect Dis J 2012; 31: 501-508.

21 Sharma D, Baughman W, Holst A, et al. Pneumococcal carriage and invasive disease in children before introduction of the 13-valent conjugate vaccine: comparison with the era before 7 -valent conjugate vaccine. Pediatr Infect Dis J 2013; 32: e45-e53.

22 Whitney CG, Farley MM, Hadler J, et al. Decline in invasive pneumococcal disease after the introduction of protein-polysaccharide conjugate vaccine. N Engl J Med 2003; 348: 1737-1746.

23 Pilishvili T, Lexau C, Farley MM, et al. Sustained reductions in invasive pneumococcal disease in the era of conjugate vaccine. J Infect Dis 2010; 201: 32-41.

24 Moore MR, Link-Gelles R, Schaffner W, et al. Effect of use of 13-valent pneumococcal conjugate vaccine in children on invasive pneumococcal disease in children and adults in the USA: analysis of multisite, population-based surveillance. Lancet Infect Dis 2015; 15: 301-309.

25 Moore MR, Gertz RE Jr, Woodbury RL, et al. Population snapshot of emergent Streptococcus pneumoniae serotype 19A in the United States, 2005. J Infect Dis 2008; 197: 1016-1027.

26 Simoes AS, Pereira L, Nunes S, et al. Clonal evolution leading to maintenance of antibiotic resistance rates among colonizing pneumococci in the PCV7 era in Portugal. J Clin Microbiol 2011; 49: 2810-2817.

27 Harboe ZB, Dalby T, Weinberger DM, et al. Impact of 13-valent pneumococcal conjugate vaccination in invasive pneumococcal disease incidence and mortality. Clin Infect Dis 2014; 59: 1066-1073.

28 Steens A, Bergsaker MA, Aaberge IS, et al. Prompt effect of replacing the 7-valent pneumococcal conjugate vaccine with the 13-valent vaccine on the epidemiology of invasive pneumococcal disease in Norway. Vaccine 2013; 31: 6232-6238

29 Waight PA, Andrews NJ, Ladhani SN, et al. Effect of the 13-valent pneumococcal conjugate vaccine on invasive pneumococcal disease in England and Wales 4 years after its introduction: an observational cohort study. Lancet Infect Dis 2015; 15: 535-543.

30 Henriqus Normark B, Christensson B, Sandgren A, et al. Clonal analysis of Streptococcus pneumoniae nonsusceptible to penicillin at day-care centers with index cases, in a region with low incidence of resistance: emergence of an invasive type 35B clone among carriers. Microb Drug Resist 2003; 9: 337-344.

31 Hermans PW, Sluijter M, Hoogenboezem T, et al. Comparative study of five different DNA fingerprint techniques for molecular typing of Streptococcus pneumoniae strains. J Clin Microbiol 1995; 33: 1606-1612.

32 Enright MC, Spratt BG. A multilocus sequence typing scheme for Streptococcus pneumoniae: identification of clones associated with serious invasive disease. Microbiology 1998; 144: 3049-3060.

33 Olsson-Liljequist B, Larsson P, Walder M, et al. Antimicrobial susceptibility testing in Sweden. III. Methodology for susceptibility testing. Scand J Infect Dis Suppl 1997; 105: 13-23.

34 Lindstrand A, Bennet R, Galanis I, et al. Sinusitis and pneumonia hospitalization after introduction of pneumococcal conjugate vaccine. Pediatrics 2014; 134: e1528-e1536.

35 Henriques B, Kalin M, Ortqvist A, et al. Molecular epidemiology of Streptococcus pneumoniae causing invasive disease in 5 countries. J Infect Dis 2000; 182: 833-839.

36 Kaplan SL, Barson WJ, Lin PL, et al. Early trends for invasive pneumococcal infections in children after the introduction of the 13-valent pneumococcal conjugate vaccine. Pediatr Infect Dis I 2013; 32: 203-207.

37 Darenberg J, Henriques Normark B. The epidemiology of pneumococcal infections - the Swedish experience. Vaccine 2009; 27: Suppl. 6, G27-G32.

38 Paterson S, Vogwill T, Buckling A, et al. Antagonistic coevolution accelerates molecular evolution. Nature 2010; 464: 275-278. 